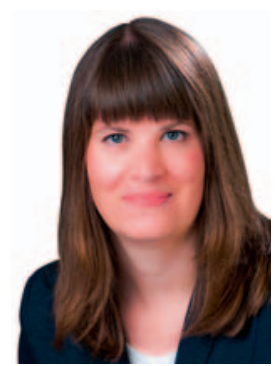

Stephanie Joachim

Koordination «OphthalmoCampus»

\section{Vorsorge treffen - für Beruf, Karriere und die eigene Gesundheit}

Die neueste Ausgabe des «OphthalmoCampus» im Karger Kompass Ophthalmologie deckt unterschiedlichste Gesichtspunkte der Augenheilkunde sowie der Aus- und Weiterbildung $a b$.

Prof. Alexa Klettner, Leiterin der Experimentellen Retinologie an der Christian-AlbrechtUniversität in Kiel, stellt uns in ihrem Beitrag einen hoch interessanten möglichen neuen Wirkstoff für die altersbedingte Makuladegeneration vor, der aus Braunalgen gewonnen wird. In einem gemeinsamen Projekt mit Kollegen aus Dänemark wird der mögliche Einsatz von Fucoidan untersucht.

Dr. Christoph Eckert berichtet über seine persönlichen Erfahrungen zur Niederlassung als Augenarzt in einer Gemeinschaftspraxis und erzählt, was ihn dazu bewogen hat sich niederzulassen und auf welche Schwierigkeiten er im Rahmen der Praxisgründung gestoßen ist. In einem weiteren Interview gibt Dr. Bernhard Mäulen, der als niedergelassener Psychiater tätig ist, Auskunft zu psychischen Erkrankungen und Suizidalität bei Ärzten. In einem Statement befasst sich die Bundesvertretung der Medizinstudierenden in Deutschland (bvmd) mit der möglichen verfassungswidrigen Vergabe von Medizinstudienplätzen. Außerdem beschäftigen wir uns mit der ärztlichen Schweigepflicht.

Bei der Lektüre des aktuellen «OphthalmoCampus» wünsche ich viel Vergnügen!<smiles>CCC1CCCCC1OC(=O)OC</smiles>

\section{KARGER}

Fax +497614520714

information@karger.com www.karger.com
() 2018 S. Karger GmbH, Freiburg
PD Dr. Stephanie Joachim

Head of Experimental Eye Research

In der Schornau 23-25, 44892 Bochum, Deutschland

stephanie.joachim@rub.de 


\section{Ophthalmologie kompakt}

\section{Fucoidan als mögliches Therapeutikum bei der altersabhängigen Makuladegeneration}

Die altersabhängige Makuladegeneration (AMD) ist die häufigste Ursache für Sehbehinderung und Erblindung in der westlichen Welt und auch in der ophthalmologischen Praxis in Deutschland allgegenwärtig [1]. Trotz aufwendiger Diagnostik, immer besserer Bildgebung und einem exzellenten Monitoring ist der Visusverlust durch die AMD nur schwer in den Griff zu bekommen. Für die Frühformen sowie die atrophische Spätform ist keine Therapie verfügbar. Und auch die Anti-Vascular Endothelial Growth Factor(VEGF)-Therapie, Standard bei der exsudativen AMD, kann einen Visusverlust nur herauszögern [2,3]. Daher sind neue und auch innovative Therapien für die AMD von großem Interesse.

\section{Wirkstoff aus dem Meer}

Eine noch wenig genutzte Quelle für mögliche neue Wirkstoffe sind die Algen der Meere. Ein interessanter Bestandteil von Braunalgen (Abb. 1) ist das Polysaccharid Fucoidan. Es ist eine heterogene Gruppe von sulfatierten Polysacchariden, die in unterschiedlichen Zusammensetzungen vorliegen, immer jedoch hauptsächlich aus Fucose mit Sulfatgruppen bestehen [4]. Fucoidane haben bereits interessante Eigenschaften gezeigt, die sie für eine mögliche medizinische Nutzung attraktiv machen könnten: So sind sie als anti-inflammatorisch, anti-oxidativ und anti-tumorös beschrieben worden [5].

Durch einen Zufall haben wir entdeckt, dass kommerziell erhältliches (heterogenes) Fucoidan in Zellen des retinalen Pigmentepithels (RPE) die VEGF-Expression und Ausschüttung reduziert. Weitere Experimente zeigten, dass Fucoidan auch die durch RPE oder VEGF induzierte Angiogenese vermindern kann. Gleichzeitig zeigte sich keine Toxizität oder Beeinträchtigung der Funktion der RPE-Zellen [6]. Für Fucoidan ist weiterhin beschrieben, dass es anti-oxidativ wirkt [7], das Komplementsystem inhibieren kann [8] und auch die Aktivierung von Makrophagen und Mikroglia reduzieren kann $[9,10]$ - alles Eigenschaften, die zumindest theoretisch der Pathogenese der AMD auch schon in den Frühformen entgegenwirken könnten [11]. Außerdem sind für Fucoidane noch weitere positive Gesundheitseigenschaften wie z.B. die Senkung des Bluthochdruckes [12] oder Übergewichtes [13] beschrieben.

Allerdings gibt es Probleme für eine mögliche medizinische Applikation von Fucoidan. Zum einen handelt es sich um ein heterogenes Polysaccharid [4], was eine Entwicklung und Zulassung für eine medizinische Anwendung schwierig macht, zum anderen haben unterschiedliche Fucoidane häufig sogar gegenteilige Ei- genschaften; so können Fucoidane je nach Herkunft, Molekulargröße und sogar Testsystem mal pro-, mal anti-angiogen wirken $[14,15]$. Daher ist eine Aufreinigung und genaue Charakterisierung von Fucoidanen unerlässlich.

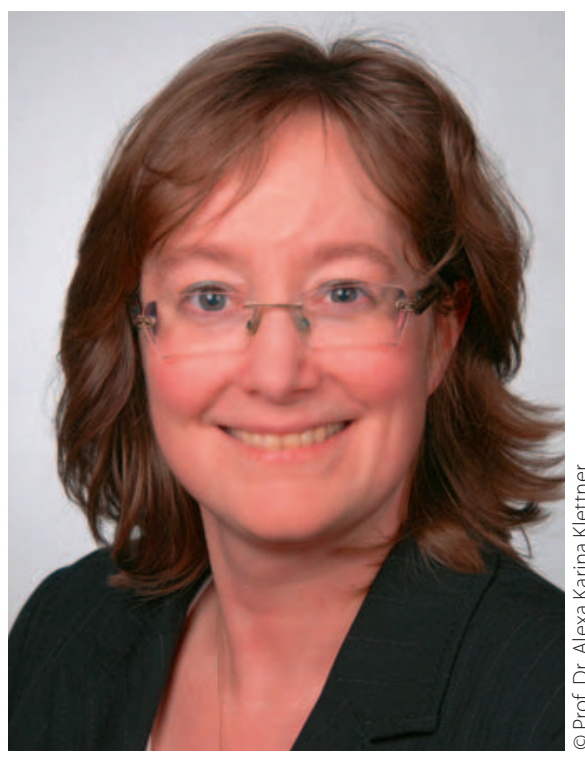

«Fucoidan besitzt Eigenschaften, ie zumindest theoretisch der Pathogenese der AMD entgegenwirken könnten.»
Noch ein weiter Weg bis zum möglichen Medikament

In dem von der Europäischen Union (Interreg Deutschland-Danmark und der Europäische Fonds für Regionale Entwicklung) geförderten Projekt FucoSan untersuchen wir in einem deutsch-dänischen Konsortium die Eigenschaften von Fucoidanextrakten. Dafür werden unterschiedlichen Algen gewonnen, verschiedene Fucoidane aufgereinigt und diese chemisch und biologisch charakterisiert. Aus diesen Extrakten werden solche mit den besten Charakteristika ausgewählt, um ihre mögliche Anwendung als Therapeutikum bei der AMD zu testen. Neben der Augenheilkunde werden in diesem Projekt auch Anwendungen in der Kosmetik und der Gewebsregeneration untersucht.

Unser Projekt steht noch am Anfang. Zurzeit untersuchen wir die ersten Extrakte in der Zellkultur. Bei positiven Ergebnissen werden wir dann zügig daran arbeiten, dieses in weiteren Modellen zu testen, um eine Reife für die ersten klinischen Studien zu erlangen. Sollten wir tatsächlich ein Therapeutikum gegen die Progression der AMD aus Fucoidan entwickeln können, könnte solch ein Medikament das Auftreten der Spätformen und so die Notwendigkeit der aufwendigen und teuren Spritzentherapie vermindern.

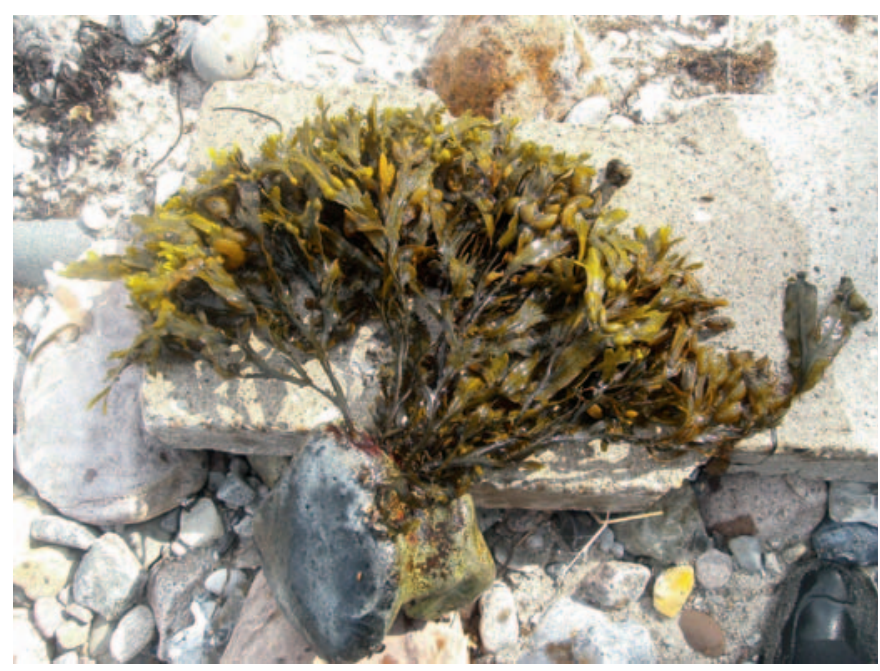

Abb.1. Braunalge der Ostsee (Blasentang). @ Marion Zenthöfer. 


\section{Literatur}

1 Schrader WF: Ophthalmologe 2006;103:742-748.

2 Schmidt-Erfurth U, et al.: Br J Ophthalmol 2014;98:1144-1167.

3 Rofagha S, et al.: Ophthalmology 2013;120:2292-2299.

4 Li B, et al.: Molecules 2008;13:1671-1695.

5 Fitton JH: Mar Drugs 2011;9:1731-1760.

6 Dithmer M, et al.: PLoS One 2014;9:e89150.

7 Marudhupandi T, et al.: Pak J Biol Sci 2014;17:402-407.

8 Tissot B, et al.: Biochim Biophys Acta 2003;1651:5-16.

9 Park HY, et al.: Food Chem Toxicol 2011;49:1745-1752.
10 Lee SH, et al.: Carbohydr Polym 2012; 89:599-606.

11 Klettner A: Mar Drugs 2016;14:E31.

12 Hernández-Corona DM, et al.: J Med Food 2014;17:830-832.

13 Wan-Loy C, et al.: Mar Drugs 2016;14:E222.

14 Pomin VH: Bioorg Med Chem 2016;24:3965-3971.

15 Dithmer M, et al.: Mar Drugs 2017;15:E193.

Kontaktadresse: Prof. Dr. Alexa Karina Klettner, Universitätsklinikum Schleswig-Holstein, Klinik für Ophthalmologie (Augenheilkunde), ArnoldHeller-Straße 3, 24105 Kiel, Deutschland, alexakarina.klettner@uksh.de.

\section{Praxisgründung und Niederlassung}

\section{Herausforderung Niederlassung - Interview mit Dr. Christoph Eckert}

Klinik oder Praxis? Das ist gar keine einfache Frage. Aber früher oder später kommt Sie doch auf (fast) jeden Arzt zu. Dr. Christoph Eckert gibt uns einen Einblick in seinen Praxisalltag und erzählt über seine Erfahrungen in der Niederlassung.

Dr. Eckert, noch vor dem Ende Ihrer Facharztweiterbildung entschieden Sie sich dafür, sich niederzulassen - was hat Sie dazu bewogen?

Meine Frau und ich hatten schon immer die Vision, Augenheilkunde auf dem höchsten Niveau in den ambulanten Bereich zu transportieren. Diese Vision haben wir nun verwirklicht.

Warum haben Sie sich für eine Gemeinschaftspraxis entschieden? Eine Gemeinschaftspraxis hat viele Vorteile. Sie können mit Kollegen auf hohem fachlichem Niveau zusammenarbeiten und gleichzeitig kommt auch die Work-Life-Balance nicht zu kurz. Das Arbeiten im Team bewirkt regen Austausch und das Arbeiten in der Praxis kann deutlich effizienter gestaltet werden.

Wie bereitet man sich auf die Herausforderung einer Niederlassung vor? Gibt es handwerkliche Grundlagen oder wird man ins kalte Wasser geworfen?

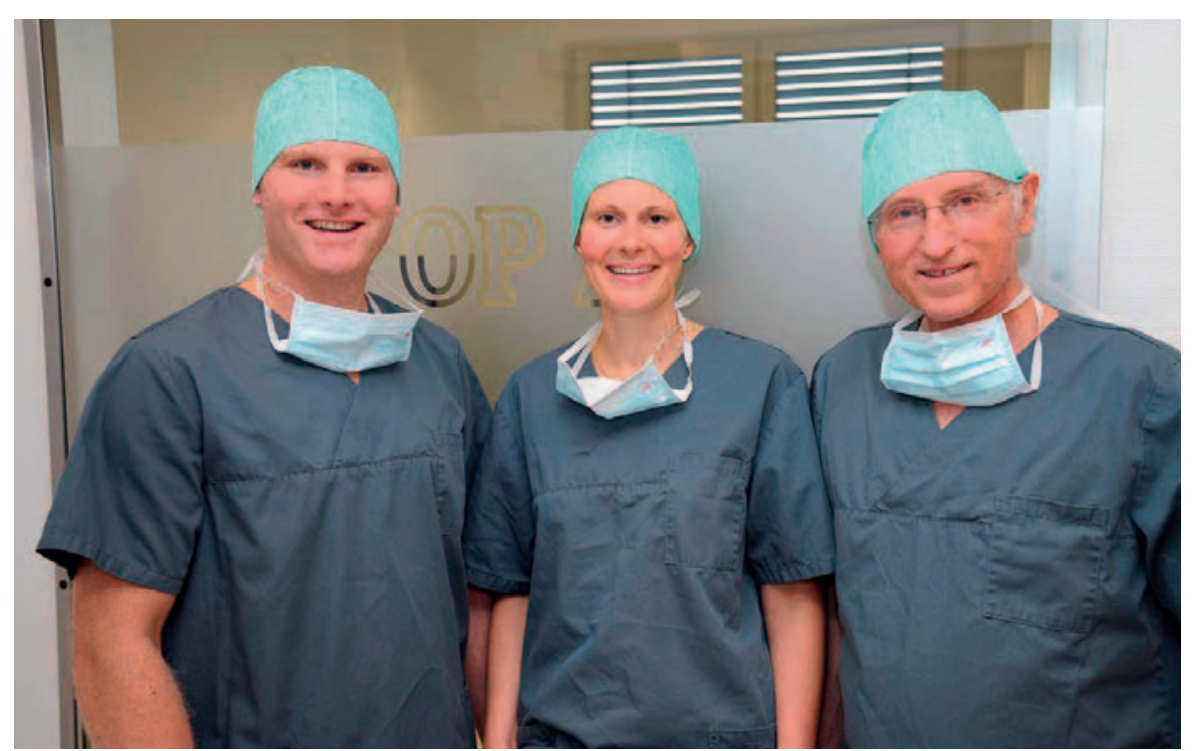

Das Augenzentrum Eckert wurde 2013 von Dr. Christoph Eckert, Dr. Sophie Eckert und Dr. Georg Eckert gegründet (v.l.n.r.). Inzwischen ist es an 20 Standorten in Süddeutschland vertreten.
Man wird definitiv ins kalte Wasser geworfen. Ein Beispiel ist die Tatsache, dass man als Operateur das erste halbe Jahr alles vorstrecken muss (sämtliche Verbrauchsmaterialien wie Linsen, OP-Material etc.). Dies wird einem erst 6 Monate später durch die KV zurückerstattet. Am Anfang ist das viel Geld, wenn man sowieso viele Schulden hat.

Ich habe über viele Jahre bis heute junge Kollegen bei der Niederlassung unterstützt. Ich war jahrelang Assistentensprecher beim Berufsverband für Augenärzte. Wenn also Kollegen Fragen haben, bin ich gerne für diese da. Inzwischen bietet aber die KV auch Beratungstermine an.

Wie gründet man eine Gemeinschaftspraxis? Welche praktischen Tipps haben Sie dafür?

Hier ist es sinnvoll, sich bei einem Rechtsanwalt für Medizinrecht Hilfe zu suchen. Denn es müssen Haftungsfragen und organisatorische Fragen geklärt werden, bevor man diesen Schritt wagt.

Zwischen 2013 bis heute haben Sie gemeinsam mit Ihrer Frau 20 Augenzentren gegründet. Sie beschäftigen derzeit über 150 Mitarbeiter. Wie kam es zu dieser erfolgreichen Entwicklung?

Qualität setzt sich durch. Immer mehr Ärzte (inzwischen ca. 40 Stück) haben sich uns angeschlossen, da wir im Augenzentrum Eckert quasi das komplette Spektrum der Augenheilkunde anbieten. Zusätzlich können die Ärzte so viel oder so wenig arbeiten, wie sie möchten. Wir haben die neuesten Geräte und sind aufgrund unserer Innovation in Deutschland sehr beliebt.

Das sieht man an der Auszeichnung, die Sie 2017 erhalten haben. Sie gehören zu den TOP 100 der mittelständischen Unternehmen. Herzlichen Glückwunsch.

Vielen Dank. Aber diese Innovation kommt nicht nur durch uns. Wir scheuen uns nicht davor, Mitarbeiter einzustellen, die besser sind als wir selbst. Nur so kommt man vor- 
an. Bei uns herrschen flache Hierarchien und die Mitarbeiter gestalten sehr viel selbst. Das ist unser Erfolgsrezept.

Ihre Ärzte sind alle sehr spezialisiert....

Ja, das stimmt. Bei uns gilt auch die Maxime: Wenn man etwas häufig und gerne macht, macht man es fast unschlagbar gut. Deshalb sind die Ärzte bei uns so spezialisiert - das Beste, was dem Patienten passieren kann.

Eines Ihrer Zentren befindet sich in Nigeria. Warum haben Sie sich gerade für dieses Land entschieden und was mussten Sie hier bei der Niederlassung beachten?

Nigeria ist ein reines Hilfsprojekt. Wir finanzieren dies komplett aus eigener Tasche. Über eine Patientin sind wir dazu gekommen. Wir bauen dort eine Augenklinik und finanzieren Ärzte und Schwestern, um den Menschen zu helfen, die die Hilfe am dringendsten brauchen. Ein tolles Gefüh!! Natürlich sind die bürokratischen Hürden in Afrika nicht zu unterschätzen. Auch Sprachbarrieren, Gefahren wie Hungersnöte und auch Terror bestimmen den Alltag.

\section{Was würden Sie für sich als Fazit nach 5 Jahren Niederlassung} ziehen?

Ich würde das sofort wieder machen. Meine Stärken sind nicht nur das Operieren, sondern auch das Organisieren. Ich bin mit Leib und Seele Augenarzt, aber auch Motivator für meine Mitarbeiter. Meine
Mitarbeiter sind meine Familie. Ich versuche jeden Tag meine Mitarbeiter zu pushen und zu innovativen Höchstleistungen anzuspornen.

\section{Was raten Sie jungen Medizinern, die über eine Niederlassung nachdenken?}

Nur Mut. Wer das Risiko eingeht, wird oft belohnt. Wer eine Art Mittelweg einschlagen will, kann sich einer Gemeinschaftspraxis, wie wir es sind, anschließen. So können auch Selbstständige im Team mitwirken, sodass man den Vorteil der Selbstständigkeit und die Sicherheit einer Anstellung hat.

\section{Dr. Eckert, vielen Dank für das Interview.}

Zum Autor

Nach dem Medizinstudium an der Universität Regensburg machte Dr. Christoph Eckert seine Facharztweiterbildung zum Augenarzt in einer Praxisklinik in Nürnberg. Schon währenddessen gründete er sein erstes Augenzentrum und wirkte an Hilfsprojekten in Äthiopien und Indien mit. Bis heute hat er gemeinsam mit seiner Frau, Dr. Sophie Eckert, 20 Augenzentren im In- und Ausland gegründet: www.augenzentrum-eckert.de.

Kontaktadresse: Dr. Christoph Eckert, Augenzentrum Eckert, Bahnhofstraße 29, 71083 Herrenberg, Deutschland, info@augenzentrum-eckert.de.

\section{Ärztegesundheit \\ Sucht, Selbstmedikation und Suizidalität bei Ärzten}

«lch werde aufmeine eigene Gesundheit, mein Wohlbefinden und meine Fähigkeiten achten, um auf höchstem Niveau zu behandeln». Dies ist eines von 13 Leitmotiven der Genfer Deklaration des Weltärztebundes und Teil der Berufsordnung deutscher Ärzte. Häufig fällt es den Ärzten jedoch schwer, ihre eigene Gesundheit bei der Pflege ihrer Patienten zu berücksichtigen. Die hohe Erwartungshaltung der Patienten aber auch sich selbst gegenüber sowie zeitliche und psychische Überlastung schlagen sich immer häufiger in seelischen Erkrankungen und Sucht nieder, die schwer wiegen und gar zum Freitod führen: Medizinerlnnen weisen empirisch belegt eine bis zu 3,4-fach höhere Suizidrate auf als die Allgemeinbevölkerung [1].

Dr. Bernhard Mäulen, Facharzt für Psychiatrie \& Psychotherapie, interessiert sich bereits seit Jahrzehnten für die seelischen Belange seiner ärztlichen Kollegen. Im Campus erörtert er die Gründe psychischer Überlastung bei Medizinern und schildert seine Erfahrungen mit Ärzten zwischen Erschöpfung, Doping und Verzweiflung. Mit umsichtigen Empfehlungen schafft er schließlich Aussichten auf Licht am Ende des Tunnels.

\section{Depressionen, Suizid und Suizidalität bei ÄrztInnen: Gab es das} schon immer?

In Ärztebiografien finden Sie immer wieder überraschende Hinweise darauf - sogar schon um das Jahr 1900. Statistisch sind Depressionen, Suizid und Suizidalität unter MedizinerInnen jedoch erst in den 1970er bzw. 1980er Jahren erfasst worden. Aber das Thema war immer da und immer schon schambesetzt. Aufgrund der Bereitschaft von US-Kollegen, sich hier zu öffnen, konnten auch bei uns betroffene Kolleglnnen anfangen, darüber zu schreiben und zu reden, was nach und nach die Größe des Problems verdeutlichte. Bei Betroffenen handelt es sich nicht um «schwarze Schafe» oder schwache Mitglieder des Berufsstands, sondern eine derartige Erkrankung trifft viele im Laufe ihrer ärztlichen Berufskarriere.

Würden Sie sagen, dass Suizidalität unter ÄrztInnen noch mehr stigmatisiert wird als in der übrigen Bevölkerung?

Ja, das würde ich. Lange hatten wir den überhöhten Anspruch: «Wenn die anderen nicht mehr können, machen wir weiter.» Gerade die «hilflosen Helfer» [2] haben Schwächen und ihre eigene Betroffenheit. Es ist ein wichtiger Teil der beruflichen Wahrheit, das anzuerkennen und offen damit umzugehen.

\section{Unterscheiden sich die Gründe für Selbstmord bei ÄrztInnen von} denen in anderen Berufsgruppen?

Die Gründe sind genau die gleichen: Depression, Suchterkrankungen, partnerschaftliche Krisen und Ähnliches; auch Psychosen, d.h. Krankheiten, die statistisch nachweisbar mit einer erhöhten Suizidalität einhergehen, treffen ÄrztInnen und zeigen die gleiche 
Symptomatik. Trotzdem gibt es eine nachweisbare, deutlich erhöhte Suizidrate bei ÄrztInnen [3].

Es gibt zwar keinen Beweis dafür, aber viele Autoren stimmen überein, dass die Fähigkeit der Helfer, selbst Hilfe anzunehmen, im Vergleich mit der Allgemeinbevölkerung weit unterdurchschnittlich ist. Dabei gilt für Depressionen wie für Sucht: Wer sich nicht behandeln lässt, wird auch nicht gesund! Außerdem sind wir nicht gerade offen auf die Betroffenen aus den eigenen Reihen zugegangen. Es brauchte Jahrzehnte, bis eine neue Kultur gewachsen ist, die das zuließ. Immer wieder erkranken die besonders Engagierten und Aufopferungsvollen, wie wir das auch vom Burnout kennen. Also, die, die angetrieben sind durch ein hohes Ich-Ideal, die sich eher wenig verzeihen, die eher selten sagen: «lch bin auch mal dran und jetzt ist hier genug».

\section{In der Ärzteschaft scheint es auffällig zu sein, dass sich besonders} viele Ärztinnen das Leben nehmen. Ist das so?

Ja, aber auch dazu gibt es keine belastbaren Daten. Für Frauen galten keine höheren Maßstäbe als für Männer. Aber Frauen waren in der Medizin lange eine Minderheit, die sich in einer Männerdomäne behaupten musste. Das hat sicherlich dazu geführt, dass mehr Biss und Härte erforderlich waren, um überhaupt Fuß zu fassen. Entsprechend schwieriger könnte es für diese Frauen gewesen sein, bei eigener Krankheit Hilfe einzufordern. Zudem kann es aufgrund der Partnerwahl dieser Frauen dazu kommen, dass sie auch zu Hause in einer starken oder überlegenen Rolle gefangen sind und aus dieser nicht so einfach aussteigen können. Aber das sind Vermutungen aus meinem Praxisalltag.

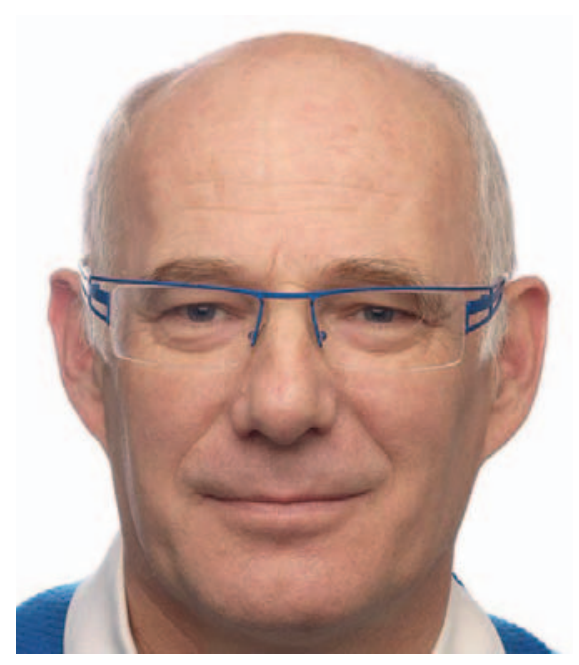

«Selbstbehandlung ist ein Kunstfehler!» gegnen und vielleicht ein Stück Ausgleich zu schaffen. Für diejenigen, die prädisponiert für eine Suchterkrankung sind, kann dies der Anfang für eine Suchtgeschichte sein, und alle Suchterkrankungen haben eine erhöhte Suizidgefahr. Bei ÄrztInnen kommt hinzu, dass sie sich zusätzlich noch Medikamente verschreiben können, die sonst nicht verfügbar sind.

\section{Ist die Situation eher ein gesellschaftliches Problem oder auch ein} Problem des Gesundheitssystems?

Es ist kein Entweder-oder, sondern ein Sowohl-als-auch. Jede/n, die/der in einer stressreichen Umgebung arbeitet, was auf den Medizinberuf zutrifft, ist der Gefahr einer Suchtkrankheit oder eines Burnouts ausgesetzt. Zudem beschäftigt sich die heutige Ärztegeneration viel ausgiebiger mit administrativen Aufgaben. Die Zeit dafür wird häufig als verloren erlebt und ruft nachhaltige Frustration und Ohnmachtserleben hervor. Zusammen mit Zeitnot ist das ein erheblicher chronischer Belastungsfaktor.

Die meisten Ärztlnnen mögen ihren Beruf, und sie werden gerne anständig dafür bezahlt - es gab Zeiten, da verdienten Mediziner weit weniger als jetzt. Die direkte Begegnung mit den Patienten ist unsere Berufung, aber die fachfremden Tätigkeiten, die bürokratischen, abrechnungsbedingten oder technischen Hintergrund haben, werden nicht als produktiv wahrgenommen, nehmen jedoch sehr viel Zeit in Anspruch. Wahrscheinlich hat die vorherige Ärztegeneration genauso viele PatientInnen behandelt und rein zeitlich genauso viel gearbeitet wie die heutige. Aber sie erlebten sich in ihrem Beruf als deutlich freier. Zwar gab es auch da ausgebrannte, suchtkranke, suizidale Ärzte, aber die aktuelle Burnout-Rate ist sehr hoch.

Betroffene MedizinerInnen nehmen deutlich weniger Antidepressiva als Betroffene in anderen Berufsgruppen, dafür deutlich mehr Psychopharmaka wie Tranquilizer, Antipsychotika - woher kommt das?

Das ist leider so. Es gibt einige Untersuchungen [4] zur Selbstmedikation bei Ärztlnnen. Bei einer Kopfschmerztablette ist das vielleicht nicht bedenklich, aber wenn es um Antidepressiva oder Antipsychotika geht, ist Selbstmedikation ein absolutes No-Go - trotzdem kommt es vor. In Peer-Überprüfungen der Selbstmedikation von Ärzten wurde die Fehlerhaftigkeit bei der Selbstmedikation belegt [5]. Es besteht das Risiko, eine falsche Substanzklasse oder Dosis einzunehmen.

Welche Rolle spielen dabei Drogen im Allgemeinen, z.B. Alkohol? «Wer Sorgen hat, hat auch Likör», hieß es früher. Alkohol als gesellschaftlich verfügbare und legitime Droge ist auch für uns Ärzte das Suchtmittel erster Wahl, um Stress, Sorgen und Überlastung zu be-
Erschwerend kommt hinzu, dass heutzutage die Krankenhäuser unterfinanziert sind und es keine geregelte Vertretung gibt: Es müssen immer alle da sein, damit der Laden läuft. Man mutet den anderen im Team mit einer krankheitsbedingten Abwesenheit sehr viel zu. Allerdings ist es paradox: Wenn ich mir beim Skifahren eine Verletzung zuziehe und erst nach zwei Monaten humpelnd wiederkomme, ist das völlig akzeptiert. Wenn ich aber wegen eines Suizidversuchs, einer Sucht oder einer Depression entsprechend lange weg bin, wird danach entweder nicht darüber gesprochen oder die Arbeitsfähigkeit in Frage gestellt. Da haben wir leider immer noch, auch unter ÄrztInnen, zwei Maßstäbe für somatische und psychiatrische Erkrankungen.

Heute fühlen sich ein Drittel der Ärztlnnen gegängelt und in einem System gefangen, das die freie ärztliche Entscheidung einschränkt. Ich glaube schon, dass dies von den äußeren situativen Faktoren mit der entscheidende ist. Aber es müssen auch individuelle Faktoren berücksichtigt werden. 


\section{Was könnte man dem Problem der Suizidalität bei ÄrztInnen konkret entgegensetzen?}

Das ist mittlerweile gut erforscht und wird bereits in der Ausbildung thematisiert: ein offener Umgang mit dem Thema, die Berücksichtigung der Stressbelastung, die eigene gesundheitliche Gefährdung, aber auch die Notwendigkeit, sich frühzeitig Hilfe zu holen. Wie bei den meisten großen Veränderungen braucht es aber fast eine Generation, bis ein Umdenken einsetzt und sich stabilisiert. Auch auf Fachkonferenzen und in der Fachliteratur wird das Thema Ärztegesundheit diskutiert. Deswegen bin ich zuversichtlich, dass die jüngeren KollegInnen theoretisch um die Gefahr wissen.

Der schwierige Faktor ist nach wie vor, ob und wann der Betroffene bereit ist, sich Hilfe zu holen. Das ist noch immer das Nadelöhr! Es gibt hier langsam einen Einstellungswandel. Aber bis betroffene ÄrztInnen tatsächlich zeitnah Unterstützungsangebote in Anspruch nehmen, gibt es noch einiges zu tun.

\section{Was würden Sie jungen/angehenden ÄrztInnen raten,} um angemessen mit psychischen Belastungen umzugehen?

Den jüngeren Ärztlnnen möchte ich sagen: Selbstbehandlung ist ein Kunstfehler! Die Medizin ist ein hochstressiger Beruf. Als angehende/r Medizinerln müssen Sie damit rechnen, dass Sie in Ihrem Beruf mindestens einmal in Ihrem Leben verklagt werden, ausbrennen oder in eine persönliche Krise geraten. Das wird die Regel sein und nicht die Ausnahme! Und es ist wichtig, dass Sie für diese Fälle vorsorgen.

Aufgrund meiner persönlichen Erfahrung würde ich das noch etwas härter formulieren: Dem System ist es ziemlich egal, wie es der/ dem einzelnen Ärztin/Arzt geht. Dir darf es aber nicht egal sein! Du bist dein erster Patient! Wieso hast du denn diesen Beruf erlernt? Gilt Mitgefühl und Fürsorge nur für die anderen? Medizinern wird der Grundsatz nihil nocere («auf keinen Fall schaden») mit auf den Weg gegeben. Wenn wir den auf uns anwenden, kann man sich fragen, ob man einen Job annehmen sollte, der uns voraussichtlich und mit hoher Wahrscheinlichkeit hoch belastet und manchmal unsere Gesundheit zerstört. Aber wenn ich mich schon fürs Arztsein entscheide, muss ich von Anfang an protektive Faktoren, einen Selbstschutz, einbauen, z.B. eine gute Ausbildung, genügend Urlaub, in Zwischenphasen gegebenenfalls ein Sabbatical, ein positives Netzwerk von KollegInnen und Unterstützern, Supervisionen, Entspannungsverfahren, und wenn nötig, hilft Coaching oder eine stützende, ambulante Psychotherapie.

\section{Dr. Mäulen, wir danken Ihnen sehr für das offene Gespräch!}

\section{Zum Autor}

Fast sein ganzes Berufsleben als Arzt hat sich Dr. Bernhard Mäulen nicht nur für das Wohlbefinden der PatientInnen, sondern auch für deren Behandler, nämlich ÄrztInnen und Therapeutlnnen, interessiert. Nachdem er sich als Psychiater niedergelassen hatte, gründete er vor über 20 Jahren die Internetseite www. aerztegesundheit.de, um die zahlreichen Anfragen von Kolleglnnen für Beratungstermine, aber auch Presseanfragen zu bewältigen und die Gesundheit von Ärzten stärker ins Bewusstsein zu rücken. Die Plattform wird heute von bis zu 100000 Userlnnen jährlich besucht.

\section{Literatur}

1 Reimer C, Trinkaus S, Jurkat HB: Suizidalität bei Ärztinnen und Ärzten. Psychiat Prax 2005;32:381-385.

2 Schmidbauer W: Die hilflosen Helfer: Über die seelische Problematik der helfenden Berufe. Reinbeck, Rowohlt, 1978.

3 Mäulen, B: Jedes Leben zählt - Suizid von Anästhesisten. Der Anaesthesist 2010:59; 395-400.

4 Montgomery AJ, Bradley C, Rochfort A, Panagopoulou E: A review of self-medication in physicians and medical students. Occup Med (Lond) 2011;61:490-497.

5 Chambers R, Belcher J: Self-reported health care over the past 10 years: a survey of general practitioners. Br J Gen Pract 1992;42:153-156.

Kontaktadresse: Dr. Bernhard Mäulen, Arzt für Psychiatrie und Psychotherapie, Institut für Ärztegesundheit, Vöhrenbacherstraße 4, 78050 Villingen, Deutschland,docmaeulen@gmail.com. 


\section{Medizinstudium \\ Urteil des Bundesverfassungsgerichts: Vergabe von Studienplätzen im Fach Humanmedizin ist teilweise verfassungswidrig - Statement bvmd}

Steigender Numerus Clausus und Wartezeiten, die länger als das Studium sind, stellen seit längerer Zeit Kritikpunkte am Zulassungsverfahren zum Medizinstudium dar. Nach der Verhandlung im Oktober verkündete das Bundesverfassungsgericht am 19. Dezember 2017 das mit Spannung erwartete Urteil bezüglich des Numerus Clausus in der Medizin. Nach Ansicht des Gerichts bedarf es Anpassungen verschiedener Aspekte des Auswahlverfahrens. Ungünstige Ortswahl darf nicht die Erfolgschancen auf einen Studienplatz reduzieren. Zudem müssen Landesunterschiede in den Abiturnoten einkalkuliert werden. Die Wartezeitquote ist praktisch nicht mehr zu halten und auch die Vergabe allein nach der Abiturnote ist nur in geringem Umfang verfassungskonform.

Doch wie soll die Studierendenauswahl in der Medizin in Zukunft aussehen?

Bereits im vergangenen Juli legten der Medizinische Fakultätentag und die Bundesvertretung der Medizinstudierenden in Deutschland (bvmd) unter Berücksichtigung des Masterplans «Medizinstudium 2020» einen gemeinsamen Vorschlag für ein transparentes Modell der Studierendenauswahl der Medizin vor. Dieser wurde nach dem Urteil an die Vorgaben des Gerichtes angepasst [1]. Im neuen Zweistufenmodell sollen neben der Abiturnote fachspezifische Studierfähigkeitstests, Situational-Judgement-Tests sowie berufspraktische Erfahrung im medizinnahen Bereich als Auswahlkriterien einbezogen werden. Die Summierung dieser vier Kriterien ermöglicht eine bundesweite Reihung. Entsprechend dieser würden 50\% der Studienplätze unter Berücksichtigung der Ortspräferenz vergeben werden. Dabei soll die Ortspräferenz wieder zu einer echten Ortpräferenz werden und nur als nachrangiges Kriterium berücksichtigt werden. Die zweite Hälfte der Plätze wird im Auswahlverfahren der Hochschulen vergeben. Dieses orientiert sich an dem ersten, bundeseinheitlichen Verfahrensschritt. Es kann aber durch individuelle Test- und Interviewverfahren erweitert werden. Eine Standardisierung, wissenschaftliche Evaluation und eine im Verlauf zielgerichtete Anpassung der eingesetzten Auswahlkriterien ist dabei notwendig.

Nach dem Urteil des Bundesverfassungsgerichtes ist nun die Politik in der Verantwortung zeitnah neue Wege für eine evidenzbasierte, faire und transparente Zulassung zum Medizinstudium zu gehen.

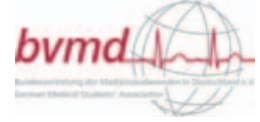

\section{Literatur}

1 Medizinischer Fakultätentag, Bundesvereinigung der Medizinstudierenden in Deutschland e.V.: Zulassung zum Medizinstudium - teilweise verfassungswidrig. medizinische-fakultaeten.de/studium/themen/aktuelle-themen/zulassung (Zugriff 08.03.2018).

Kontaktadresse: Carolin Siech, Bundesvertretung der Medizinstudierenden in Deutschland e.V. (bvmd), Robert-Koch-Platz 7, 10115 Berlin, Deutschland, pr@bvmd.de.

\section{Medizinrecht \\ Ärztliche Schweigepflicht: Wann Ärzte Auskunft erteilen dürfen - und wann nicht}

Liegt eine gültige Schweigepflichtentbindung des Patienten vor, können Ärzte einem Betreuer oder der Polizei Auskunft erteilen. Ohne diese Entbindung ist die Rechtmäßigkeit sorgfältig im Einzelfall zu prüfen.

Im ärztlichen Alltag fallen viele hochsensible Daten an. Ein diskreter Umgang mit diesen Daten ist daher notwendig, um das Vertrauensverhältnis zwischen Arzt und Patient zu gewährleisten. Dies hat einen derart hohen Stellenwert, dass es durch § 203 des Strafgesetzbuchs und das medizinische Standesrecht geschützt wird. Bereits das Gelöbnis, das zu Beginn der Muster-Berufsordnung für die in Deutschland tätigen Ärztinnen und Ärzte abgedruckt ist, greift die ärztliche Schweigepflicht auf. Jeder Arzt hat die ihm «anvertrauten Geheimnisse auch über den Tod der Patientin oder des Patienten hinaus» zu wahren. Die Verpflichtung zur Verschwiegenheit ist jedoch nicht immer so einfach einzuhalten. Es gibt durchaus rechtlich legitimierte Ausnahmen, die ein Brechen der ärztlichen Schweigepflicht erfordern.

\section{Anfrage des Vormundes oder Betreuers}

Immer wieder erreichen Ärzte Anfragen mit der Bitte um Auskunft oder gar Akteneinsicht. Verlangt der Patient selbst Einsicht in seine
Krankenakte, muss ihm dies in der Regel ohne Einschränkung gewährt werden, wenngleich er idealerweise in einem Arzt-PatientenGespräch Einsicht nehmen sollte. Auch wenn ein gesetzlich bestellter Vormund oder Betreuer mit diesem Anliegen an den Arzt herantritt, hat er ein Recht auf Akteneinsicht. Ist beispielsweise ein

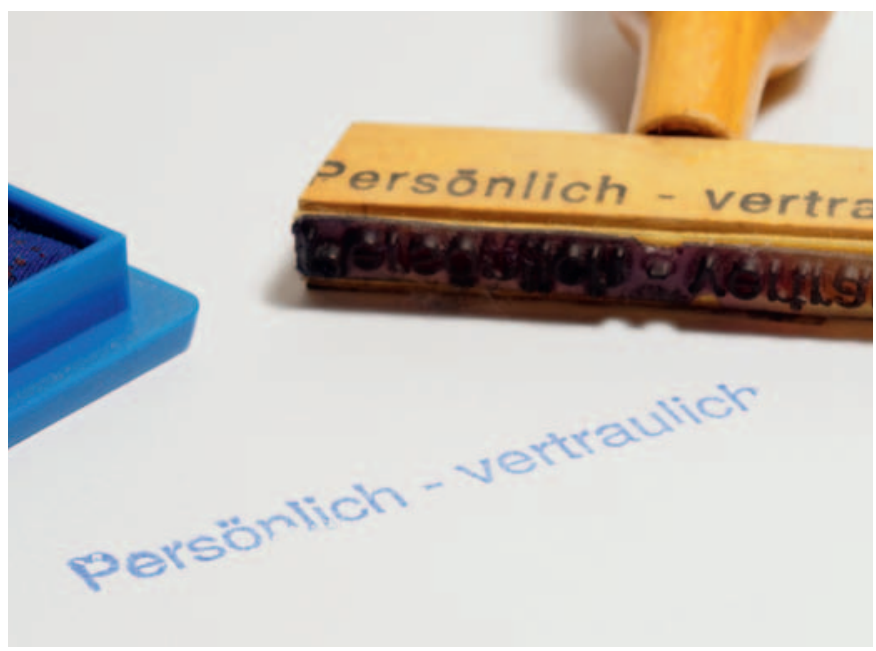


Volljähriger aufgrund einer psychischen Krankheit oder einer körperlichen, geistigen oder seelischen Behinderung nicht in der Lage, seine Angelegenheiten ganz oder teilweise zu erledigen, kann das Betreuungsgericht einen Betreuer als gesetzlichen Vertreter bestellen. Die Betreuung ist immer auf jene Aufgaben beschränkt, in denen sie erforderlich ist. Mögliche Aufgaben sind Vermögensfürsorge, Postkontrolle, Aufenthaltsbestimmungsrecht oder Gesundheitsfürsorge. Ein Betreuer hat nur Recht auf Auskunft oder Akteneinsicht beim Arzt, wenn die Auskunft für die Wahrnehmung seiner konkreten Aufgabe nötig ist und zwar nur im erforderlichen Umfang. So benötigt ein Betreuer im Aufgabenkreis Vermögensfürsorge beispielsweise keine vollumfängliche Einsicht in die Patientenakte. Das Recht auf Auskunft eines Betreuers kann zusätzlich durch den Willen des Patienten eingeschränkt werden, sofern er einsichtsfähig ist. Eine Offenbarung von Patientengeheimnissen gegen den Willen eines einsichtsfähigen betreuten Patienten ist nicht zulässig. Daher sollte jede Anfrage eines Betreuers vorab sorgfältig geprüft werden.

\section{Anfrage von Ermittlungsbehörden und Gerichten}

Anfragen von Ermittlungsbehörden oder Gerichten erzeugen meist eine besonders hohe Unsicherheit. Man möchte einerseits keine personenbezogenen Daten unbefugt offenbaren, gleichzeitig jedoch die Ermittlungsarbeit der Behörden, wie die Suche nach vermissten Personen oder Unfallopfern, nicht behindern. Grundsätzlich folgt aus der strafrechtlich fixierten ärztlichen Schweigepflicht (§ 203 StGB) ein Zeugnisverweigerungsrecht für Ärzte sowie deren Berufshelfer (§53 Abs. $1 \mathrm{Nr} .3$ und $\S 53 \mathrm{a} \mathrm{StPO}$ ) gegenüber den Ermittlungsbehörden und vor Gericht.

Das im November 2015 in Kraft getretene Bundesmeldegesetz berechtigt Krankenhäuser jedoch dazu, gegenüber den zuständigen Behörden Auskunft in bestimmtem Umfang zu erteilen. Dieser umfasst Vor- und Familiennamen, Geburtsdatum und -ort (bei Geburten im Ausland auch den Staat), Staatsangehörigkeit, Anschrift sowie das Datum der Aufnahme und der Entlassung. Die Krankenhäuser dürfen Auskünfte jedoch nur erteilen, wenn dies zur Abwehr einer erheblichen und gegenwärtigen Gefahr, zur Verfolgung von Straftaten oder zur Aufklärung des Schicksals von Vermissten und Unfallopfern erforderlich ist. Den konkreten Grund für das Auskunftsersuchen muss die anfragende Behörde im Einzelfall darlegen. Eine vom Patienten verfügte Auskunftssperre greift in diesem Fall nicht. Jedoch dürfen Ärzte darüber hinaus auch gegenüber der Polizei, Staatsanwaltschaft oder den Gerichten keinerlei Auskünfte über Patienten erteilen. Anders verhält es sich, wenn der Patient den Arzt von der Verpflichtung zur Verschwiegenheit gegenüber der Polizei oder der Staatsanwaltschaft entbunden hat. Dann müssen Ärzte Auskunft erteilen.
Sie ist jedoch auf den in der Entbindungserklärung festgelegten Umfang zu beschränken. Schweigepflichtentbindungen von Angehörigen sind grundsätzlich unwirksam, selbst wenn der Patient bereits verstorben ist. Dennoch können Angehörige und Erben nach $\S 630 \mathrm{~g}$ Abs. 3 BGB ein Recht auf Einsicht in die Patientenakten haben.

\section{Wenn Gefahr im Verzug ist}

Das Zeugnisverweigerungsrecht wird durch das Beschlagnahmeverbot ( $\$ 97 \mathrm{StPO}$ ) auf alle Aufzeichnungen, Mitteilungen und Gegenstände der ärztlichen Untersuchungsbefunde erweitert, die sich im Gewahrsam des Krankenhauses befinden. Dazu zählt auch die Patientenakte. Als Folge darf das Krankenhaus Unterlagen nur herausgeben, wenn entweder der Patient eine Entbindung von der Schweigepflicht vorgenommen hat oder die Ermittlungsbehörden einen Beschlagnahmebeschluss vorweisen, den in der Regel nur ein Richter ausstellen kann. Ausnahme: Wenn durch den Zeitverlust beim Einholen einer richterlichen Genehmigung Beweismittel verlorengehen können, ist «Gefahr im Verzug». Dann sind auch Polizei oder Staatsanwaltschaft berechtigt, eine Beschlagnahme anzuordnen.

Liegt ein gültiger Beschlagnahmebeschluss vor, müssen die Unterlagen im Original herausgegeben werden. Dabei gilt, unbedingt den genauen Umfang der Anordnung zu beachten, also auf welche Unterlagen sich diese konkret bezieht. Um die Patientensicherheit zu gewährleisten, sollten von allen Dokumenten Kopien angefertigt werden, bevor die Unterlagen herausgegeben werden. Zusätzlich sollten im Beschlagnahmeverzeichnis alle herausgegebenen Dokumente vollständig aufgeführt sein.

\section{Widerspruch gegen die Beschlagnahme}

Wenn die Ermittlungsbehörden keinen Beschlagnahmebeschluss vorlegen können, sollten Krankenhausmitarbeiter der Herausgabe ausdrücklich widersprechen und auf eine Dokumentation des Widerspruchs im Beschlagnahmeprotokoll bestehen. Fordern die Behörden dennoch die Herausgabe der Unterlagen, muss das Krankenhaus die Beschlagnahme dulden. Die Mitarbeiter sollten den Beamten dann lediglich zeigen, wo die Unterlagen lagern, diese jedoch nicht aktiv herausgeben.

\section{Originalquelle}

Kropp H, Günther U: Ärztliche Schweigepflicht: Wann Ärzte Auskunft erteilen dürfen - und wann nicht. Dtsch Arztebl 2017: 114; 48. (Direktlink zur Originalversion: www.aerzteblatt.de/archiv/195023/Aerztliche-Schweigepflicht-Wann-Aerzte-Auskunfterteilen-duerfen-und-wann-nicht)

Kontaktadresse: Dr. sc. hum. Uwe Günther, Sanovis GmbH, Richard-StraussStraße 69, 81679 München, Deutschland, info@sanovis.com. 